\title{
Achieving Diagnosis by Consensus
}

Received: date / Accepted: date

\begin{abstract}
This paper provides an analysis of the collaborative work conducted at a multidisciplinary medical team meeting, where a patient's definitive diagnosis is agreed, by consensus. The features that distinguish this process of diagnostic work by consensus are examined in depth. The current use of technology to support this collaborative activity is described, and experienced deficiencies are identified. Emphasis is placed on the visual and perceptual difficulty for individual specialities in making interpretations, and on how, through collaboration in discussion, definitive diagnosis is actually achieved. The challenge for providing adequate support for the multidisciplinary team at their meeting is outlined, given the multifaceted nature of the setting, i.e. patient management, educational, organizational and social functions, that need to be satisfied.
\end{abstract}

Keywords Diagnosis · ethnography · healthcare · interaction analysis · medical diagnosis $\cdot$ multidisciplinary medical team meetings $\cdot$ technology teleconference

\section{Introduction}

This paper provides an analysis of the diagnostic work of the multidisciplinary medical team (MDT) at their meeting (MDTM) and the use of technology in the MDTM setting, in order to inform the design of technology to support the MDTM and MDTM-like activities, to contribute to our un-

B. Kane and S. Luz

Department of Computer Science

Trinity College Dublin, Ireland

Tel.: +353-1-896- $\{2381,3686\}$

E-mail: \{kaneb,luzs\}@cs.tcd.ie 
derstanding of the nature of diagnostic work, and provoke insights into the design and use of technology to support collaboration in this setting.

Medical work that concerns making a diagnosis is usually associated with an individual clinician, such as a local family, or hospital, doctor. In some cases, such as the work of a pathologist, diagnostic work is glamorised in media as being akin to detective work, and it shares resemblance in many respects. For all diagnostic work, information and facts are gathered, preliminary interpretations are made and refined as more information becomes available, usually for the purpose of taking some action or making changes. Like any scientific study, attributions are made to explain the facts observed, and will likely be influenced by our understanding of the underlying processes and our social and political circumstances.

Despite public perception that a diagnosis is made by an individual, almost all diagnostic work is collaborative in some way, by its nature. Berg (1992) argues that a physician, in transforming a patient's problem into a solvable problem, does not simply combine some cognitive items together, such as historical and examination data, but actively articulates an array of heterogeneous elements within the transformation. Berg observes that 'locally situated routines' form the frame of reference for medical action, and that biomedical knowledge alone does not shape clinical practice (Berg 1992).

Rather than the traditional approach in studying diagnostic work from the perspective of an individual diagnostician, and how he/she makes a decision, this study examines how the MDTM diagnosis is collaboratively achieved. This paper focusses on a work meeting situation where diagnoses are made by a group of clinical specialists, by consensus. Each speciality brings a particular perspective, or professional vision (Goodwin 1994) on the problem, (a socially organised way of seeing and understanding events), and an agreed diagnosis is achieved through dialogue. This setting is the multidisciplinary medical team meeting (MDTM) for patient management, a forum that has become routine in health care over recent years. The MDTM is a collaborative forum, and provides a useful model for the study of diagnostic work. In Berg's parlance, it is a forum where medical action is facilitated by allowing the articulation process to be performed and where, simultaneously, action is defined as proper, or not, in the circumstances. While all diagnostic work can be considered to involve collaboration and information sharing among specialists, often asynchronously, the MDTM is a particular formal occasion for synchronous collaborative diagnostic work. At the MDTM, data presentation, analysis and interpretation are negotiated interactively and an awareness of the outcome is shared by all of the team.

It will be explained how the accuracy of the patient diagnosis is improved, and articulation is achieved, in collaboration through talk among clinical specialists at MDTMs. Attention is also being drawn to how technology can further improve the diagnostic performance and also how the misuse of technology, or inappropriate technology, can undermine the effectiveness of the meeting.

The problem of diagnosis is often linked directly with the decision on treatment. For the purposes of this paper, the remedial action, or treatment decision, that might be taken as a result of a diagnosis is not addressed in 
any detail. The focus in this paper is on the process of making a definitive diagnosis by a group, through collaboration, that is achieved at an MDTM (as opposed to a clinical / pathological / radiological diagnosis made by an individual specialist). The interactions involved in making a patient management decision are quite different and will only be referred to in passing, in the context of how the decision on the remedial action task might differ from the diagnostic task. By concentrating on how the diagnosis is accomplished in the MDTM setting, allows for a greater understanding of the nature of diagnostic work conducted through collaboration among specialists.

The structure of this paper is as follows. A background explanation of the logic for the MDTM as a diagnostic tool will be given followed by a brief history of the development of the MDTM as a forum for definitive diagnosis, by consensus. The methodology used in this study will then be summarised followed by a description of how the diagnosis is actually achieved in the MDTM process. The account of proceedings at an MDTM when making a diagnosis includes the use of artefacts, and use of communication technology, by the participants. While the MDTM utilises teleconferencing technology to extend its services to remote locations, this paper will not address the specific issues on the use of teleconferencing technology, in detail. Attention will be drawn to problems introduced by teleconferencing technology, in a more general way, in the context of a technology that is regularly used during MDTMs and facilitates interaction over geographical distance. The use of artefacts and gestures is considered separately. A discussion follows that highlights the benefits and challenges in the application of technology into this setting and, finally, recommendations will be made that have the potential to make the MDTM more effective.

\subsection{Rationale for diagnosis at MDTMs}

The method of a definitive diagnosis being made by a group, through collaboration, (sometimes referred to as 'triple assessment'), is a relatively new idea and likely to become in more widespread use in the future. Traditionally an individual patient was managed by an individual clinical consultant, and while this is still the situation medico-legally with an individual clinician being responsible for any patient discussed at an MDTM, we are witnessing a shift towards more team responsibility for individual patient care (Kane et al. 2008). Evidence suggests that diagnoses made by consensus achieve a higher performance than diagnoses made by an individual clinician (Weller and Mann 1997; Wit 1998). Decision-making by a group, i.e. diagnosis by group consensus, is conceptually very different from traditional models of making a diagnosis. Instead of the tradition model, described by Cicourel (1990) for example, where an individual clinician makes a diagnosis, following receipt of information and collaboration from distributed, independent, sources, the MDT decision making involves several different specialists contributing information to the group in real time. The group collectively synthesise the information contributed by individual team members, reconcile any inconsistencies and agree a diagnosis. 
Medical diagnostic work involves investigating a phenomenon, i.e. the patient's problem. The tools available for investigation utilise a range of technologies, such as fiberoptics, dye chemistry, and x-rays, to gather facts appropriate with our models of disease processes. There is no perfect test. Instead, we are presented with a range of tests that report a complex set of performance indices: sensitivity, specificity, likelihood ratios, positive predictive values (PPV), etc. (Gallagher 2003). Some general rules apply in medical work, namely, to use the test that provides the greatest possibility of giving the correct answer (Guyatt et al. 2000), with the least invasive procedure for the patient, and to select tests appropriate to the clinician's hypothesis, or hunch. To investigate if the leading hypothesis is true, the test is selected with high specificity to confirm the leading hypothesis and a test with a high sensitivity is chosen to exclude an alternative hypothesis (Richardson et al. 1999). Typically in medical investigations, three separate disciplines are utilised, namely clinical observation, pathology laboratory testing and radiological imaging. While none of these specialities alone will guarantee a $100 \%$ accuracy, with minimal invasion of the patient, a combination of tests will yield a satisfactory probability level to proceed with management. Continuing audit and review, as in evidence-based medicine, will refine guidelines for the investigation of different diseases.

To complicate matters, many of the individual investigative tests are not quantitative measurements and involve qualitative assessment. For example a chest x-ray: the interpretation of the result of the procedure is a matter of professional judgement. While affected by sampling and technical methods (including "artefacts", e.g. image distortions), pathology and radiology opinion is subject to the shortcomings that can bias interpretative tasks. Although training and professional experience can demonstrate high task performance, an individual can ignore, misunderstand, or misinterpret data. It is also possible that an individual may be too economical in collecting data, or for a clinician to become psychologically committed to a particular hypothesis, making it more difficult to restructure a problem (Elstein and Schwarz 2002). Collaborative work structures where multidisciplinary teams operate, have the potential to improve individual work practices. However, they are often poorly supported by technology as will be illustrated in this paper.

\subsection{Development of MDTMs}

The MDTM developed from its role in teaching under-graduate and postgraduate medical students and has its origins in clinical-pathology, clinicalradiology and surgical risk management meetings (Wong and Birks 2004). It provides a forum for the introduction to new concepts and techniques in both clinical and experimental medical sciences and has evolved in response to the increasing complexities of patient care and demands for improved quality of services. Within multidisciplinary medical teams (MDTs) each specialist role contributes his, or her, particular skill and knowledge with respect to the patient's condition. From the patient's perspective, the MDTM facilitates their review and advice from the multidisciplinary team. For the hospital, the 
group engages in peer review, and the forum provides potential for on-going learning and education. MDTMs provide clinicians with exposure to a range of clinical experience which would be difficult to acquire otherwise, such as cases that are uncommon, involve difficult decisions, or that present specific diagnostic dilemmas. From the organizational viewpoint, the MDTM facilitates the collection of information for audit purposes, it serves quality process objectives and allows for comparison between different investigative procedures in the diagnostic task. The MDTM thus contributes information for the development of quality processes, including clinical practice guidelines, and can be regarded as an organizational quality mechanism.

Over recent years there has been significant growth in multidisciplinary team working (Ruhstaller et al. 2006), because of increasing specialisation, advances in medical technologies (Batchelor and McFarlane 1980), including teleconferencing, and recommendations by respected agencies (Wright et al. 2007; Board of the Faculty of Clinical Radiology 2005; NCCAC 2005; Rasmussen and Bulow 2005; Alberts et al. 2003; Calman and Hine 1995). These developments have led to a need for highly specialised health professionals to engage in intense collaboration to provide effective services (Arnaudova and Jakubowski 2005; Hall and Weaver 2001) and building multi-disciplinary (or interdisciplinary) teams to address the complex problems arising in patient care can reduce health care system error (Øvretveit 1999).

While this paper focusses on the diagnostic work of the MDTM, the educational role of multidisciplinary team meetings is long recognised and the practice is well developed within university teaching hospitals (Wright et al. 2007). Approaches to information sharing through mutual activity in narrative is considered efficient (Haidet and Paterniti 2003), and a powerful learning tool (Mott et al. 1999). Apart from its traditional education and audit function, the practice of having MDTMs for routine patient management has become increasingly necessary and MDTs and their meetings now occupy a central role in developed health systems (Houssami and Sainsbury 2006). Patient outcomes have been shown to benefit from having their treatment managed in a MDT setting (Birchall et al. 2004; Sainsbury et al. 1995) and multidisciplinary team (MDT) working is now advised as a better way of organising health services for patients, particularly cancer patients (Wright et al. 2007; NCCAC 2005). MDT practice has become widespread, particularly in the United Kingdom, and MDTMs are being incorporated as a standard into cancer patient care pathways in Europe as well as in Australia and North America (Nicholls 2007; Alberts 2007; Balding and Anderson 2007; O'Higgins 2006). As the benefits in cancer care are being appreciated, MDTMs are being advocated as the standard of care for a wider range of illnesses (Windsor and Forbes 2007; Jefford et al. 2007). The practice of multidisciplinary medical team meetings is expected to grow in coming years as the demand for additional meetings, where more patient cases can be discussed in greater depth, is realised.

Although advocated by many agencies as an improved method of working, and the mutual activity in building narrative is agreed to be an efficient method of information sharing, it is acknowledged that there is little empirical evidence for this recommendation (National Collaborating Centre for 
Acute Care (NICE) 2005). While many guidelines acknowledge the difficulties in making a definitive diagnosis and refer to 'triple assessment' (O'Higgins 2006), it should be pointed out that many of the guidelines place emphasis on the treatment benefits identified as a result of MDTM discussion. Cancer treatment can be complex and involve either surgery, chemotherapy and / or radiation oncology administered either singly, or in combination. Combined treatments can be administered in sequence, or concurrently, and the co-ordination of such services can pose challenges for clinical administration.

\section{Methodology}

The field study reported here draws upon CSCW approaches to analysing work, and to articulating ways that team members collaborate. The general ethnomethodological approach is that advocated by Randall et al. (2007) in that ethnography is used as a method to understand the real world characteristics and context of the work where technology is used (or might be used). Randall et al. (2007) argue that success in design is associated, in complex ways, with the social context of system use. Observing interactions within their natural setting provide insights, and sensitivities, that are valuable in informing system design.

This observational methodology is employed here to shed light on an aspect of the work of a multidisciplinary team meeting that was revealed as part of a larger, and on-going, study. While the collaborative diagnostic work that takes place at an MDTM is central to this study, the tasks and information generation, information flow and handling, leading up to the meeting are considered critical to the task and are taken into account. Therefore, although the data provided for this paper derived mainly from observation of MDTMs over a prolonged period, sometimes the observational work prompted a need for more structured investigation of specific aspects of MDTM work. When this happened, semi-formal descriptive methods such as the task analysis techniques discussed below and qualitative data gathering methods were employed which complemented and often sharpened the observational research.

Observing the work of a multidisciplinary team (MDT) was conducted by the first author as a non-medical researcher and team member, over approximately 4 years. This researcher had prior experience of working with the MDT as a medical scientist, which facilitated her integration into the team and understanding of the diagnostic context of the interactions at the MDTM. Several exercises were undertaken and video recordings were made to gather specific data and verify observations. Specific exercises included i) a questionnaire investigating the audio and visual needs of the participants during discussion and when in teleconference, ii) a set of evaluation exercises conducted over a number of meetings that sought to identify specific elements of the MDTM that are more satisfactory than other parts, iii) an exercise to assess information sharing during discussion and iv) an exercise undertaken to assess team members' ability to follow discussion. While these exercises are reported in detail by Kane and Luz (2006b), aspects that concern the diagnostic work at the meeting are re-examined for the purpose of 
this paper. Informal and semi-structured interviews were held with each of the participant roles from time to time over the course of this study.

The identified structure in a patient case discussion (PCD), defined as discussion stages (D-Stages) in this study, utilises the foci for analysis of Jordan and Henderson (1995), combined with task analysis approaches of Pinelle et al. (2003), Paternò (2000) and van der Veer et al. (1996) and is described in Section 4. The observational data and interviews also served to provide task analysis of the MDTM and associated work processes. Adapted task diagrams are included in this paper that illustrate the two identifiable stages in the PCD that encompass the collaborative diagnostic task. Analysis of the tasks conducted through interactions during a PCD borrows ideas from Pinelle et al. (2003) on the 'mechanics of collaboration' which examines the teamwork that goes on in a group task (represented graphically in Figures 3 and 4). Ideas are also drawn from the 'Concurtask trees' of Paternò (2000) who recognises that activities may not follow a strict linear process and examines concurrent events and activities in the execution of the task.

The video recordings were examined using the general approach of interaction analysts (Jordan and Henderson 1995) which examines the human activities in the interaction, in the context of the tasks (van der Veer et al. 1996) and information flow. This approach proved particularly useful in identifying the participation structure through which the definitive diagnosis is accomplished.

Although the methods used are presented in order, results informed one another and there was an interplay between the research processes employed. We believe that the methods complimented one another and help present a comprehensive account of the collaboration achieved in dialogue. All examples given in this paper are real, and anonymised to protect patient privacy.

\section{The Diagnostic Process}

Before describing the MDTM, it is important to appreciate the pre-MDTM work that leads up to the MDTM and the 'outcomes' which serve as 'inputs' to the MDTM discussion. The MDTM represents a coalescence of independent, but related, work activities. The pre-MDTM work undertaken provides important context for the MDTM discussion, without which the function of the MDTM cannot be fully appreciated. The narrative that follows will explain the function of the MDTM in the overall patient management process and will provide a reference for later discussion. The example used is that of a respiratory MDT. The same process applies to other groups but the detail will differ, depending on the organs involved.

Suppose a patient, Jane Doe, feels generally unwell, and goes to her family doctor (GP), who talks to her, elicits her experienced complaints (symptoms) and makes observations (signs), facilitated by the use of a stethoscope. The GP anticipates that a referral to a respiratory physician may be appropriate and orders a plain chest radiograph $\left(\mathrm{CXR}^{1}\right)$ from a local service.

\footnotetext{
1 Chest radiograph, or chest x-ray, is an examination of the chest using x-rays. It is a routine investigation in patients with query of lung or heart disease.
} 
A couple of days later, the GP receives a written report of Jane Doe's CXR that says there is evidence of 'consolidation in the left lower lobe of the lung'. Since this sounds like the patient would benefit from specialist input, the GP requests a hospital out-patient (OPD) appointment with a respiratory specialist.

Following a short time-frame (perhaps a couple of weeks, depending on urgency and demand), the respiratory physician meets Jane Doe in OPD, talks to her about her symptoms, conducts a physical examination and decides to 'have a look' with a fibreoptic bronchoscope which allows examination of the air-ways and facilitates sampling for pathology. A repeat CXR is ordered, a $\mathrm{CT}^{2}$ scan, and perhaps a $\mathrm{PET}^{3}$ scan, depending on the intuition of the respiratory physician. A PET scan demonstrates areas of abnormal cellular activity in the body and is useful when assessing a patient for metastatic tumour deposits. Although sensitive, a PET scan is not $100 \%$ specific. Areas of inflammation, such as a damaged rib, will have increased uptake of $\mathrm{FDG}^{4}$ and may be over-interpreted as 'positive for tumour'. However, a negative PET scan is usually a pre-requisite for thoracic surgery and supports a decision to remove a lung tumour, since removal of the affected lung is ill advised if there are numerous secondary tumour deposits. In conjunction with diagnostic imaging studies, other tests are also ordered such as pulmonary function and blood tests (including clotting factors), and a bronchoscopy is scheduled - likely within days.

When Jane Doe arrives for bronchoscopy, in a day or two, her results are checked and, unless there is a problem with her clotting factors or some other serious reason, she receives sedation and the bronchoscopy procedure goes ahead. The respiratory physician can visualise the airways and observe any abnormality in colouring, texture or physical distortion and takes samples for pathology (brushing of the surface, biopsies, and washings of the area). Jane Doe is tabled for discussion at the following Monday morning's MDTM.

Every Friday, each team member receives a list of patients that have been tabled for discussion for the following Monday morning. The list will comprise all new cases for initial assessment, recurring patients who need to be reassessed as well as any patient demonstrating complications and requires multidisciplinary team review of their case. Jane Doe is a new patient and on the list circulated. Her samples are reviewed by specialist pathologists and radiologists, along with the other patients tabled for discussion at the next MDTM. The CXR that the GP arranged is located and reviewed in conjunction with recent imaging and any other imaging studies available.

None of these specialities (clinical, radiology or pathology) expect to be always able to identify lung cancer. A shadow on a CXR could represent a number of different diseases, either benign or malignant; a negative CXR does not necessarily mean that the patient does not have a tumour. Sen-

\footnotetext{
${ }^{2}$ computed tomography, is a computer analysis of a series of x-ray pictures which allows a 3-dimensional construction to be made from the series.

3 positron emission technology is a specialised imaging technique that uses shortlived radioactive substances (radioisotopes) to produce 3-dimensional coloured images of those substances functioning in the body

${ }^{4}$ F-18 fluorodeoxyglucose activity on positron emission tomography. FDG is a radioactive sugar and the most common radioisotope used in PET scanning.
} 
sitivity, specificity rates and $\mathrm{PPV}^{5} \mathrm{~s}$ are usually cited for each modality of investigation. For example, a transbronchial needle aspirate is expected to yield a positive result in approximately $42 \%$ of positive cases (Chin et al. 2002) and while is not a very sensitive test, it can be very useful if positive, since invasive surgery for a tissue sample can be avoided for 4 out of 10 patients. Generally speaking for investigative tests, the lack of sensitivity and specificity is attributed to a) technical limitations of the method, b) human errors in sampling, c) technical error in processing and d) human interpretation errors.

However, at the MDTM, when the 3-way correlation of results takes place, the sensitivity and specificity of the work process is increased and the diagnosis is made more accurately than any of the individual methods alone will allow. This is called the 'definitive diagnosis' as distinct from the 'clinical diagnosis', 'radiological diagnosis' or 'pathology diagnosis' that may be contained within the written reports from results of the respective procedures. The definitive diagnosis, if cancer, is presented as a 'disease stage' classification. Combining independent investigations, each of which has its limitations, is generally accepted to improve the overall investigative performance (Genest and Zidek 1986). The following thought experiment is used by Hogarth (1986) to illustrate the point: Would you have more or less faith in a medical diagnosis if there was agreement between two experts using different methods of diagnosis as opposed to agreement between two experts using the same method?

Furthermore, group decision making, by consensus, improves the performance of the investigation. Weller and Mann (1997) demonstrate that majorities invariably 'do better' than individuals, and thus, it is reasonable to assume that the diagnosis agreed at the MDTM, by consensus, has a higher accuracy both because of the nature of the interactions at the meeting, when individual professional opinion is refined and modified, and also because of the number of people involved in the decision.

\subsection{TNM disease staging}

Cancer classification and staging allows a physician to determine treatment more reliably, to evaluate results of management more accurately and to compare worldwide statistics (AJCC 1992). Correct disease staging is critically important for appropriate patient management (Cicourel 1990) and is one of the key items of information generated through collaboration at the MDTM.

The currently accepted staging method used is called 'TNM staging', where $\mathrm{T}$ refers to the tumour size, $\mathrm{N}$ to lymph node involvement by tumour and $\mathrm{M}$ is for distant metastases ${ }^{6}$, or secondary tumours. A tumour that is classified as a T2N1Mx represents a tumour over a certain size $(3 \mathrm{cms}$ for lung in 2008), there is some local lymph node involvement and the question of whether, or not, there are metastases has not yet been determined. The

\footnotetext{
5 Positive Predictive Value

6 Metastases represent secondary cancer deposits that have travelled a distance from the primary cancer site via lymph or blood channels
} 
anatomic extent of the disease provides the primary basis for staging. The histopathologic ${ }^{7}$ grade and age of the patient are factors in some tumours. It is likely that developments in molecular biology and proteomics research will play a part in disease staging, or classification, in the future.

Clinical, radiological and pathological staging are conducted independently and recorded as distinct entities within reports issued from clinical assessment, radiology reports and pathology reports. The definitive TNM staging system provides a consistent and reproducible description of the extent of anatomic involvement (Lababede et al. 1999) and is arrived at through a combination of clinical findings, radiological imaging and histopathological diagnosis. Having the TNM disease stage agreed by consensus at the MDTM is considered to have a higher status than a TNM stage proposed by a single speciality. If there is a conflict between what is written in a pathology report for example, with the TNM stage agreed at the MDTM, the records from the MDTM discussion is considered to be the significant record for clinical audit (Hollywood 2007).

The TNM system is used for all lung cancers except small cell lung cancers (SCLC), which are staged separately. For SCLCs the most important categorisation is whether the tumour is 'limited stage' or 'extensive stage'. TNM staging can be difficult to determine at times, and there are some situations in which additional investigations will be undertaken before the TNM stage is finally established. Sometimes, for example, it might be difficult to differentiate between a T1N1 and a T4 in a situation with an apparent small tumour on histopathology (a T1) with possible 'widespread lymphatic involvement' on radiology (which would define it as T4). There are situations from time to time when a patient may have two primary tumours, both of which are $\mathrm{T} 1 \mathrm{~N} 1$, and very difficult to distinguish from a T4 with 'widespread lymphatic involvement'. The treatment approach and prognosis is very different for the two scenarios.

TNM also has a system of sub-classification: a pre-treatment clinical classification (cTNM or TNM) and a post-surgical histopathologic pathological classification (pTNM). There is also a 'yTNM' representing the disease stage, post-treatment with chemotherapy, or radiation therapy. All classifications are retained unaltered in the patient's record.

While pathological diagnosis is usually considered to be the 'true' assessment (because it is determined by direct examination of the tumour), it is only possible with early stage disease, or post-mortem. Clinical staging uses indirect observations of a tumour, but is often the only means available. Clinical decisions are guided by the clinical staging for advanced disease. The cTNM will influence the possible selection for adjuvant chemotherapy and is used as an estimation of prognosis. Pathological staging is most valuable in early stage disease. The TNM classification replaces another staging system called, Stage 1, Stage lla and llb, Stage llla and lllb and Stage IV. It is common practice for both categorisations to be used when discussing cases, but it is the TNM staging that is considered the one on which guideline and treatment decisions are based.

\footnotetext{
${ }^{7}$ Histopathology is the branch of pathology that studies disease changes at tissue structure level
} 
The identification of the correct TNM category, is often not straightforward and a decision support tool to assist the MDT in determination the TNM stage would be a useful reference guide for the MDTM, and is currently under development. Figure 1 summarises the categorisation for lung cancer staging as an example of how the category is determined. Definitions such as the TNM staging for lung cancer illustrated in Figure 1, are continually under review and development.

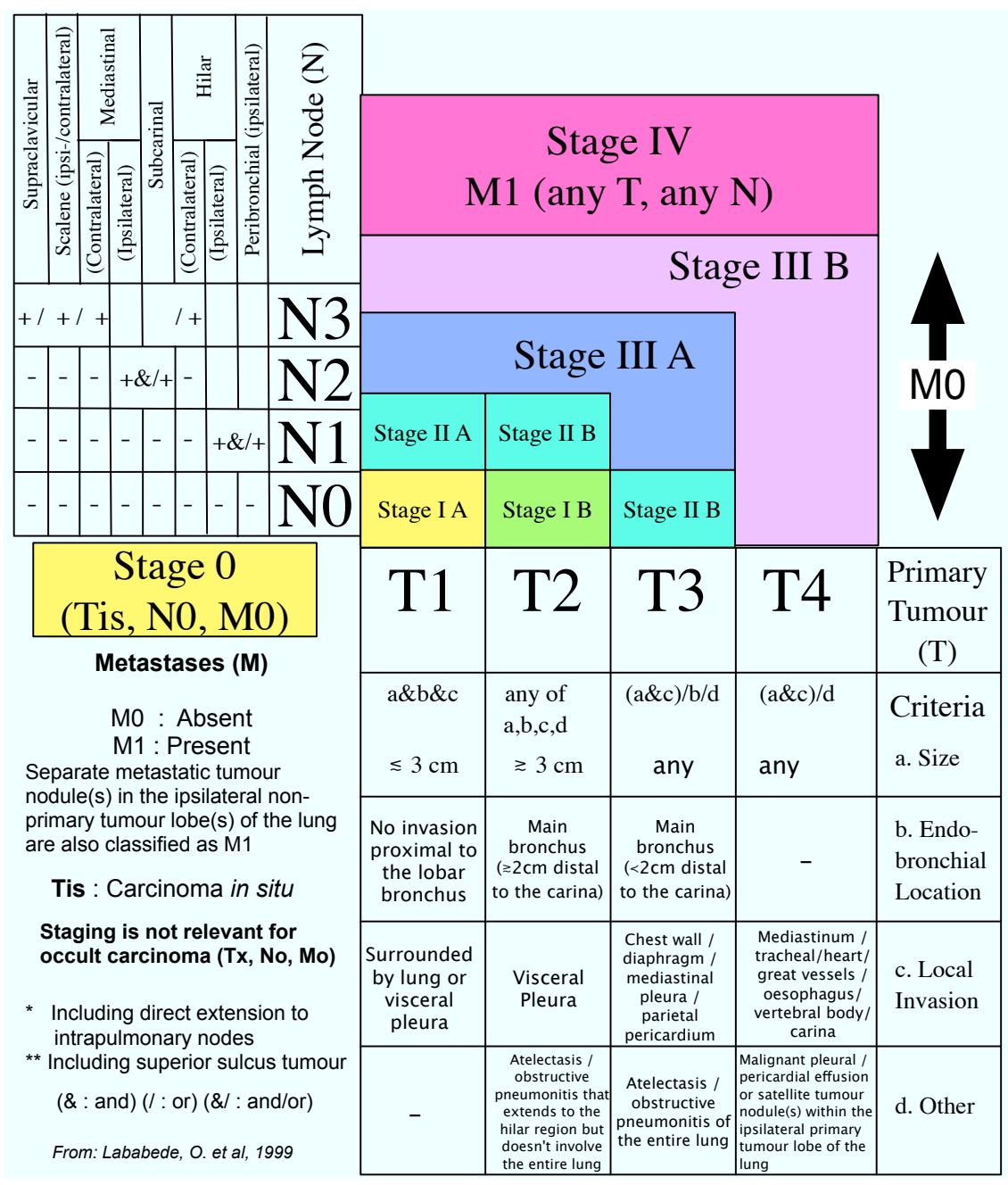

Fig. 1 TNM staging of Lung Cancer, from Lababede, O. et al, 1999

Continuing with the story of Jane Doe. The results of each of her investigations are presented to the MDTM (following pre-MDTM review), it is agreed that her tumour stage is T1N0M0, and it is decided to recommend 
that she should have surgery to remove the affected lobe of lung. But since she didn't have a PET scan, she will have one done. Unless it is positive, she will be scheduled for surgery and reviewed again at the MDTM after her operation. Her PET scan is clear, so she proceeds with surgery. Following surgery, the lung segment removed is sent to pathology for processing and microscopic examination. She has follow-up imaging conducted and is tabled for re-discussion at the MDTM, allowing 5 working days for the tissue removed to be processed, stained with dyes and examined microscopically.

Like the pre-surgical discussion, her pathology and radiology is reviewed separately in each department, re-presented to the MDTM, discussed, and a post-treatment disease stage diagnosis is agreed. It is decided to send her for some chemotherapy/ radiation therapy and she will be re-evaluated at a future MDTM.

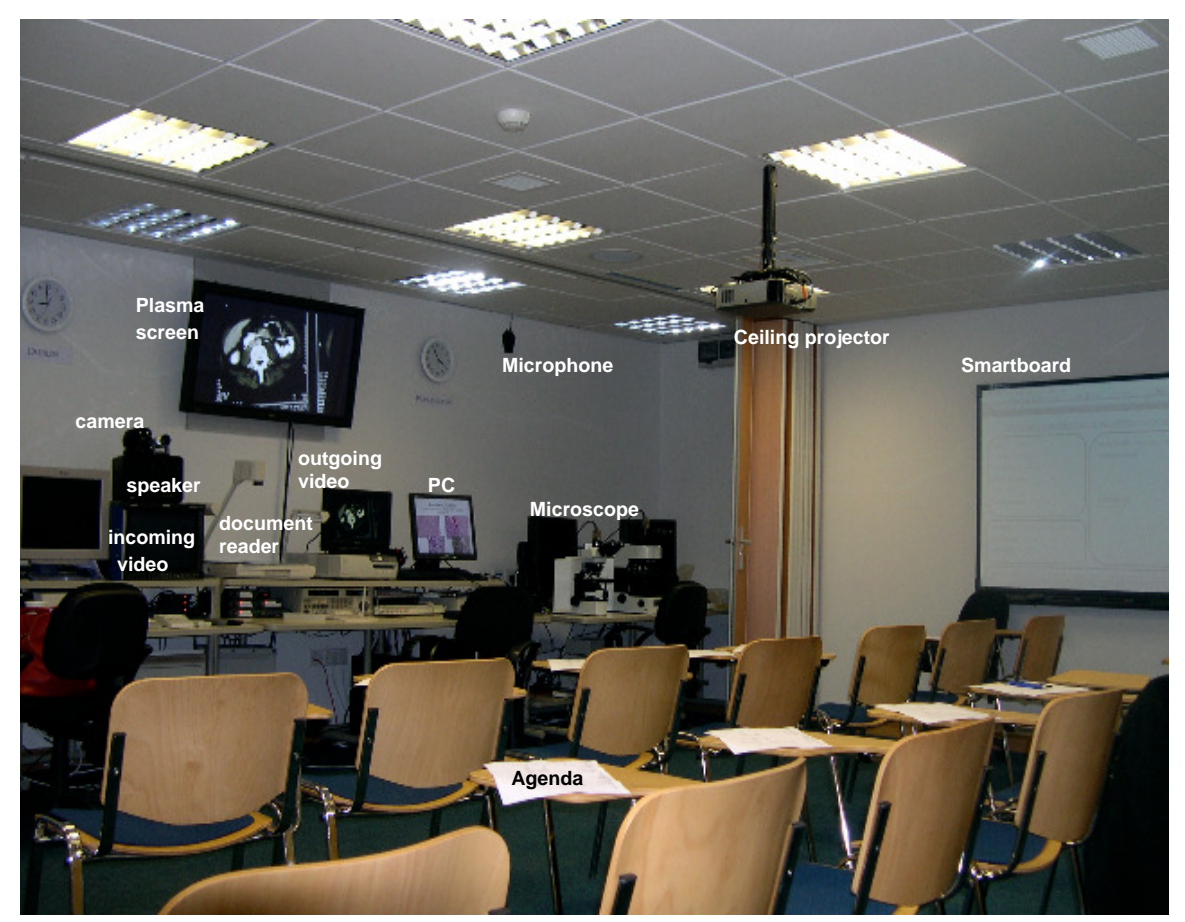

Fig. 2 The technology used at MDTM includes a microscope, document reader, PC, SmartBoard ${ }^{\mathrm{TM}}$ and plasma screen display. Participants face forward, towards the large plasma display. Facts are summarised in the text display on side-wall.

\section{Diagnosis at the MDTM}

In this section the collaborative task of making the definitive diagnosis, that is achieved through interaction among the specialists, during a patient case 
discussion (PCD) is examined in detail. Terminology used to describe MDT meetings can differ, depending on the geographic location, or biologic sitespecific emphasis, but the practices observed are broadly similar. Terms used include 'tumor boards' (Katterhagen and Wishart 1977), 'consensus meetings' (Groth 2008), 'surgical risk management meetings' (Wong and Birks 2004), 'multidisciplinary cancer conferences' (Wright et al. 2007) and 'multidisciplinary care' meetings (Li et al. 2008). Patient case discussions can be expected to have general similarities to the description provided here, while exhibiting some local variation. Typically, teams meet weekly for one to two hours and participants are primarily representatives from medical, surgical, medical oncology and radiation oncology, pathology, diagnostic radiology, palliative care, nursing and allied professionals such as physiotherapists and speech therapists (Ontario 2008). About 30 people are in attendance at the meetings described here, which is typical (Ontario 2008). Smaller hospitals or highly specialised groups may have fewer people in attendance and these may be connected by communication technology such as video conferencing (Li et al. 2008). Medical students and medical staff undergoing specialist training are also in attendance. Specialist trainees at MDTM are normally employed as registrar members of the team and present details of any individual patient to whom they have been assigned. In the earlier stages of MDTM development, clinically 'interesting', or complex cases were tabled for discussion. However, in compliance with current clinical practice guidelines, all cancer patients, or patients suspected of having cancer, are tabled for discussion. In practice, non-cancer patient cases that are complex or have unusual presentation are included on the agenda, such as T.B. or sarcoidisis. It is the responsibility of the clinician responsible for an individual patient to ensure that their patient is tabled for MDTM discussion.

The mechanics of the collaborative task are represented graphically in Figures 3 and 4, borrowing ideas from Pinelle et al. (2003). Explicit communication in spoken, written and gestural messages are identified within the diagnostic task during a PCD. Whenever possible, attention is drawn to mechanics of information communication such as awareness, feedback, visual evidence and overhearing (Pinelle et al. 2003). Ideas are also drawn from the 'concurtask trees' of Paternò (2000). Most of the tasks conducted in a PCD are performed in sequence. Arrows and integers in the diagrams, indicate the temporal relationships between events. Integers are used to indicate sequential activities and the use of the same integer points to tasks that can be done concurrently. Recursion is represented using double-headed arrows and concurrent tasks are described within each phase of the discussion, where appropriate. Solid lines represent links between events that always occur, while broken lines signify events that are often, but not always, observed.

The structure of events has been described by Jordan and Henderson (1995) as stretches of interaction that cohere in some manner that is meaningful. Easily identifiable behavioural units within such structures are called 'ethnographic chunks'. The identification of such chunks is regarded as a first step towards analysis. In this study of diagnosis by collaboration in PCDs at MDTMs, structures, or stages, are observed which are being called D-Stages. 
As well as the discussion stages (D-Stages) of PCDs at an MDTM, the beginnings and endings of MDTMs are collaborative achievements and worthy of note as they reveal the sorts of props and technologies that are thought to be necessary to carry off the event (Jordan and Henderson 1995), such as the diagnosis task in a PCD. Before the start of the MDTM, the equipment, shown in Figure 2, is checked and made ready for use, namely the microscope, document reader, $\mathrm{PCs}(2)$, an overhead display and a SmartBoard ${ }^{\mathrm{TM}}$, all of which are used in the diagnostic process described here. The pathologist uses the microscope to demonstrate tissue samples. The radiologist uses PCs to show electronic radiological imaging studies, and the document reader to project radiological imaging studies on plain film. The document reader is also used occasionally by medical staff to show printed images taken at bronchoscopy. The SmartBoard ${ }^{\mathrm{TM}}$ on the side wall was installed to facilitate lectures in the room but was adopted by the team for MDTM use. Information pertinent to the patient under discussion is projected onto the SmartBoard ${ }^{\mathrm{TM}}$, (described in more detail later on page 30). A laser pointer is also available; a device mostly used by the pathologist when illustrating features in tissue biopsies. The radiologists tend to use an index finger for pointing when they use the document reader, and they use the mouse as a pointing device when using the PC. The technology in Figure 2 also incorporates a teleconferencing system, called Telesynergy ${ }_{\circledast}$, of which the cameras, a microphone, speaker and in-coming and out-going video stream displays are shown. The Telesynergy $\circledast_{\circledast}$ system facilitates the team to be extended (i.e. distributed) over multiple sites.

The internal structure of the PCD is being defined here in discussion stages, called D-Stages, and is analogous to the term 'segmentation of events' used by Jordan and Henderson (1995). These D-Stages are identified though review of video recordings of PCDs and while there are 4 D-Stages identified within a discussion, concentration here is on the first two D-Stages which concern the diagnosis task. The remaining two D-Stages concern the patient treatment decision that is made once the definitive diagnosis (TNM stage) is established, and while the treatment decision, or patient management plan, is referred to it will not be discussed here in any detail.

\subsection{D-Stage 1: first part of the case discussion}

The opening of a case discussion determines the start of D-Stage 1. The patient's initial presenting symptoms and clinical findings, (contained in a printed page that is circulated on the Friday prior to the Monday meeting), are reported verbally by a registrar. Typically the recount by the registrar is in a narrative style, providing minimal information at first and revealing more information as necessary during discussion, or as required by other participants. Example opening:

"Jane Doe is a 36 year old woman who presented to her GP feeling unwell. She has a persistent cough and shortness of breath for the past few months. She is an ex-smoker with a 30 pack year ${ }^{8}$ his-

\footnotetext{
81 packet of cigarettes per day for a year is defined as a ' 1 pack year'
} 
tory. On examination finger clubbing was noted. There was no major adenopathy".

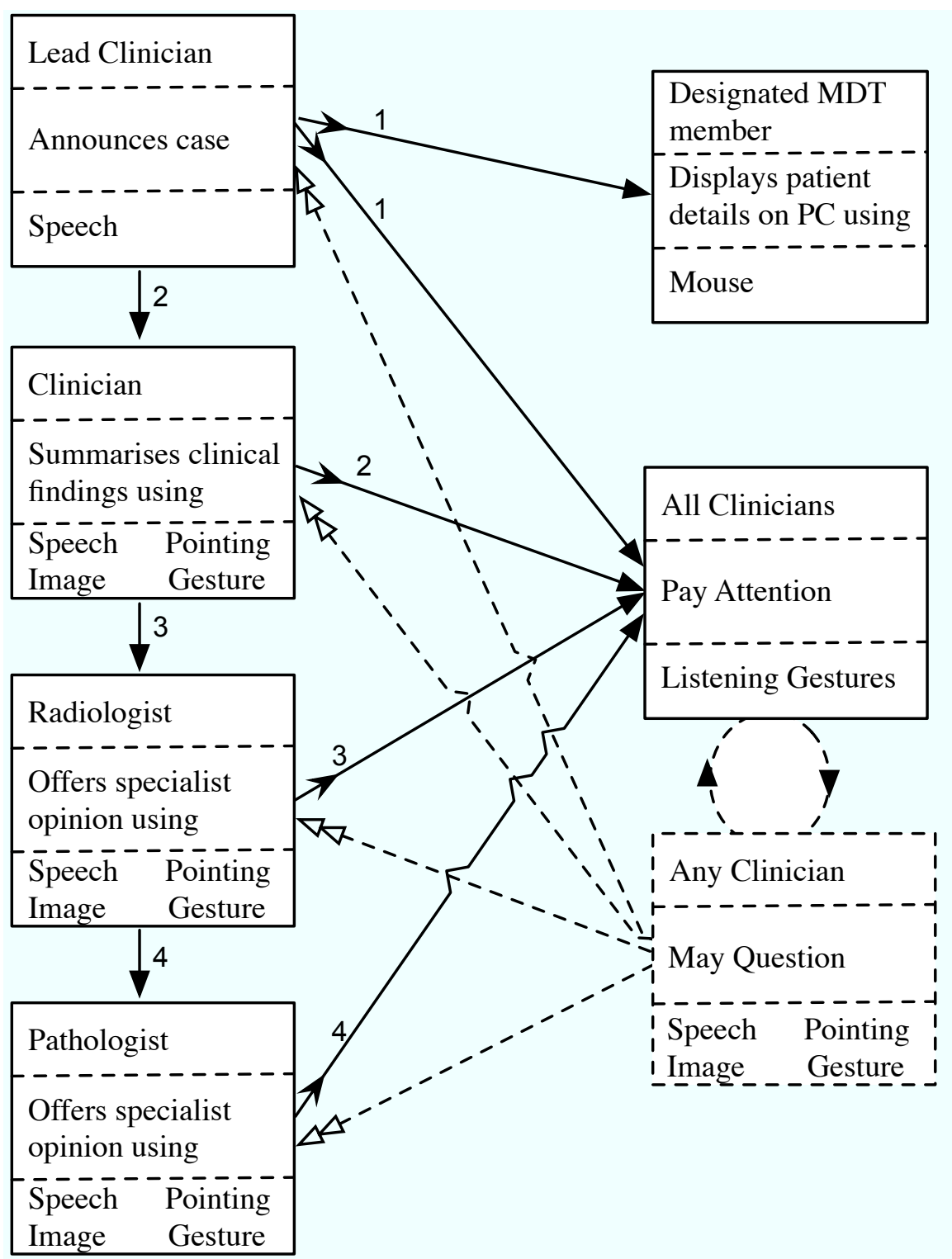

Fig. 3 D-Stage 1

The patient's story unfolds as a mystery writer might tell a story: the signs, symptoms and findings presenting clues and allowing the attendees to speculate on the differential diagnoses as the story unfolds. Thus, a partici- 
pant may (privately) guess what might be coming next and solve the problem before the entire story is revealed, or revise their initial hunch on the basis of additional information. Generally, people consider the most common possibilities in the first instance, while keeping an open mind about unusual possibilities, as data are gathered by the group in discussion.

After the registrar has summarised the initial presenting symptoms, the team member who conducted the bronchoscopy (bronchoscopist) continues the story:

"At bronchoscopy the airways looked a bit inflamed. We didn't see any tumour, but took washings for cytology and a transbronchial biopsy from an area in the left lower [lobe of lung] that looked a little narrowed."

If the bronchoscopist feels that he/she may want to discuss the visual appearance of the airways later, video and digital camera equipment are available during the procedure for image capture. At the discretion of the bronchoscopist, colour images from the procedure, printed on paper, may be projected for the group to visualise (or passed among senior staff) at the MDTM. Following requests from MDT members, the endoscopy system can now capture images electronically and have them accessible across the network, for review during MDTM discussion.

Following the clinical information being presented (that includes a report on any bronchoscopy procedure), the radiologist continues the narrative while presenting images and highlighting the important features on the projected image using a pointer. The radiologist when showing the images will also relate the findings in a narrative, and in time sequence. First, old images are viewed, if available. These are followed by the chest x-ray and the CT scan. The PET scan is then shown if available. The radiologist might say, for Jane Doe:

"I have a radiograph here from her GP [pointing to, and outlining, the left lower lobe shadow] ... its fairly well demarcated, about $1 \mathrm{~cm}$ in diameter ... and another [removes cxr film from the document reader and proceeds to load another image on PC from the hospital PACS ${ }^{9}$ ... taken last week, ... you can see here [pointing] that it has increased in size ... [pointing to, and outlining, the left lower lobe shadow again] ... it looks more like ... $1.5 / 2 \mathrm{~cm}$... and there's a 7 week interval inbetween ... so it seems to be getting bigger. We have a CT as well and you can see [as he closes the image on screen and opens another image file from the $P A C S]$... that ... its much easier to see ... and its close to the pleura ... we don't seem to have a PET. Was one ordered?"

The registrar replies that the PET wasn't ordered yet, and the co-ordinator takes a note in anticipation. This is a task that will probably need to be done once the MDTM is over (but has not yet been agreed to be necessary).

The radiologist continues, when demonstrating features in an image:

"It looks like a mass to me ...maybe a T1. I can't see any nodal involvement ... a PET will help with that. Of course, it could, maybe, be a bronchocoele ... maybe ... but I think its more likely tumour".

\footnotetext{
${ }^{9}$ Picture Archive and Communication System
} 
The pathologist picks up the story and, sitting at the microscope connected to the large plasma screen display, shows first the cytological washing that has some inflammatory cells only, highlights notable features with a laser pointer, and continues:

"The transbronchial [biopsy] contains a moderately differentiated adenocarcinoma that is TTF $^{10}$ positive. Do we know that there's no history of any previous adenocarcinoma?"

The registrar responds: "She was never in hospital before now."

Occasional questions are asked of the presenters, by the observing members of the team during the presentation of findings that seek more information, or confirmation, on the presenting symptoms recounted such as:

"Is her ECOG really 1?"

"What's her occupation?", or,

"How long since she's given up smoking?".

From time to time, during co-located discussions only, it has been observed that a senior member of the team might speculate on the final diagnosis (prematurely, and with good humour), or might ask observers to speculate on the diagnosis given the clinical story and the radiology picture only. If someone is proven wrong subsequently, it is not a cause of embarrassment or censure, but if they are proven correct it is greeted with good humoured praise or awe. Teleconference discussions tend to be a little more formal than co-located discussions.

More questions may be asked of the individual presenters, such as:

"And you didn't see anything in the right [bronchus]?".

Some discussion on these findings usually follows by the consultant staff and differential diagnoses are postulated:

"Do you think this could be sarcoid? Or what about T.B?"

Facilitating mutual activity, as in the narrative account of patient history, can provide important insights (Haidet and Paterniti 2003). The presentation of the patient's clinical history to the meeting does this efficiently without requiring large expenditures of time.

Figure 3 is an adapted task diagram to represent the roles involved, the activities and interactions in D-Stage 1. Each rectangle represents an event. Within each rectangle the three sub-divisions describe the role(s), action and method used to conduct the task. (Explanation is given in page 13.)

D-Stage 1 closes following the presentations of the initial facts of the case, or the start of the next D-Stage.

\subsection{D-Stage 2: Second part of the case discussion}

The start of D-Stage 2 is determined by the first question following the presentations in D-Stage 1. The clinical correlation of the pathology, radiology

\footnotetext{
10 TTF-1 is an immunochemical cell marker that is usually positive in lung (as well as some other) tumour cells
} 
and findings on examination, presented in D-Stage 1, characterises D-Stage 2. The findings that were presented in D-Stage 1 are considered in relation to one another and in relation to the patient's general state of health and clinical presentation. Findings from the 3 specialities should be concordant, with one another. Questions are frequently asked, and clarification sought, by team members. This part of the meeting is important in capturing unsatisfactory events in the individual diagnostic processes. As already explained, neither radiology nor pathology nor clinical examination can consistently diagnose disease and demonstrate $100 \%$ sensitivity and specificity. All have the potential to give wrong results. In pathology, for example, errors may be caused through sampling, artefacts in processing tissue, or interpretative error.

The outcomes of the different investigations should be compatible, or any inconsistencies easily explained. Reviewing the findings of several independent investigations together, at the meeting, reduces the possibility of a patient being wrongly assessed. Lack of concordance, warrants discussion and explanation. Both false positive and false negative results, are likely to be revealed when examined in the context of the information provided by related investigations. Katterhagen and Wishart (1977) explain that even the most careful and well-informed physician may occasionally make errors in judgement, overlook significant aspects of a case, forget an important detail or be unaware of a new development. When a case is analysed by several physicians, each from his/ her own point of view and each with learning experiences unique to his/ her speciality, the chances of omission or error are greatly reduced. Furthermore, the opportunities for the interjection of new knowledge are increased. In other words, by each of the specialists' professional opinion being presented for peer review, and open to real time modification and interpretation in the context of the other specialists' opinion, the diagnostic sensitivity and specificity of the diagnostic process are increased over the sensitivity and specificity for any of the single modes of investigation (such as pathology or radiology).

An example of an exchange in D-Stage 2:

A clinician might say:

"Yes, that [pathology] picture fits with the CT".

Or the radiologist might say:

"Even if pathology says its not tumour, I still think there's tumour there and you must have missed it" [referring to the clinician who took the pathology sample].

In the case of Jane Doe, the radiologist would likely revise his/her original interpretation of the image, and say on seeing the pathology:

"OK, I take back what I said about the bronchocoele . . it's obviously tumour."

and the pathologist might challenge radiology and say:

"Are you sure that's the primary? Is there any possibility we're looking at a met ${ }^{11}$ here."

11 'met' is used here as short for metastases 
to which the radiologist would reply:

"I didn't see any sign of anything else - and I don't have any other imaging. An abdominal scan would be useful - but the PET should sort that one out - so we don't need an abdominal scan."

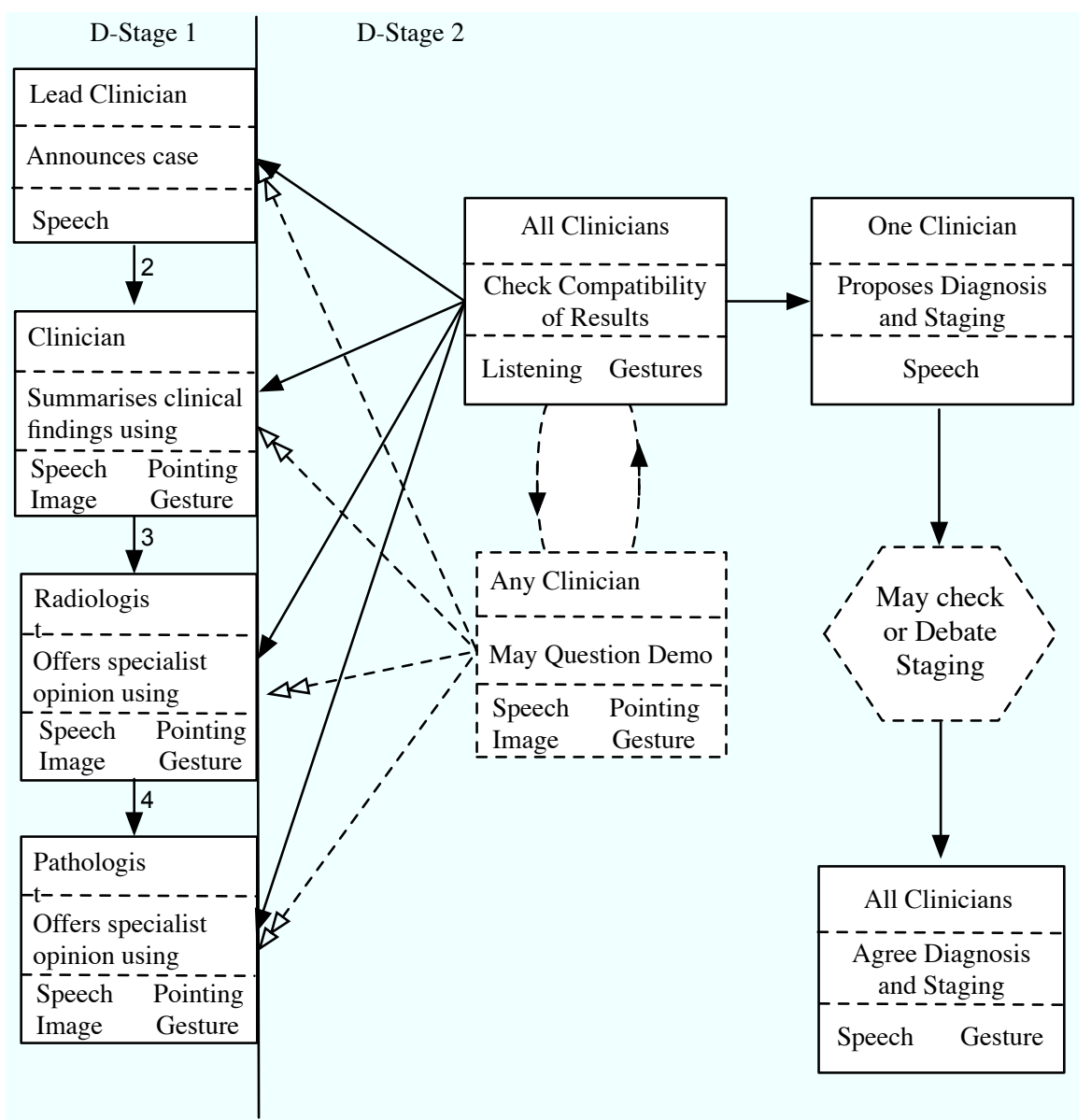

Fig. 4 D-Stage 2

The aim at this stage of the proceedings is to agree a definitive diagnosis and establish the disease staging. By the end of D-Stage 2 the patient's presenting symptoms have been reviewed and the patient's diagnosis has been established. The combined information of the clinical radiological and pathology findings provides the information for the patient's disease stage using the TNM classification (Figure 1). Jane Doe is determined to be a T1N0M0, pending a satisfactory (negative) PET scan.

The cancer patient's disease staging is the main factor (but not the only one) that influences the choice of treatment approach, and informs the dis- 
cussion on management options in the latter part of the PCD. (The other factors influencing choice of treatment are the patient's level of fitness and any coexistent disease.)

The presentation of the detail of the patient case, and questioning afterwards, may sometimes reveal some of the difficulties and underlying stress for clinicians when dealing with people in tragic circumstances on a daily basis. It has been observed that a clinician, when presenting a case, may be hoping that the finding is really benign. In the case of Jane Doe, it might be said:

"are you sure its tumour - any chance there was a mix up with the specimens in the lab? She's only 36 - she has 4 young children and her husband was recently diagnosed with heart disease."

Such negotiations have also been observed by Hardstone et al. (2004) in inter-service work where various membership categories will determine if a patient fits referral criteria and what help can be offered. Stories are told and scenarios developed that involve formulations about the categories to which patients may be assigned, such as the example in page 14. In Jane Doe's case, she is categorised as a relatively young female with a significant smoking history, even though she is an 'ex-smoker'. While she exhibits signs of having a chronic disease (finger clubbing), she does not have enlarged lymph nodes (lymphadenopathy) which excludes her from a particular set of diseases. Figure 4 represents the actions that may take place in D-Stage 2. Not all PCDs have a D-Stage 2 . Sometimes, when the clinical data are clearly presented and the radiology and pathology are straightforward, there is no need for further discussion and the PCD will proceed directly to the patient management decision in D-Stage 3. An example of classical pulmonary Tuberculosis (T.B.) is a case in point. Once the classical caseating granuloma with multinucleated giant macrophages and $\mathrm{ZN}^{12}+$ (positive) mycobacteria are demonstrated on histopathology and the classical radiological and clinical signs are present, the discussion proceeds directly past D-Stage 2 to confirm the treatment protocol. In cases of T.B. there is a recognised treatment protocol, so, unless other individual patient circumstances complicate the management, there is a clear treatment guideline to be implemented.

\subsection{Structures}

A typical PCD does not take as much time as one might imagine from this account. The duration for a case discussion can range from 3 minutes to 20 minutes, or longer, depending on the patient's complications and the finding from the investigations.

However, PCDs held across a teleconferencing link have already been shown to take significantly more time than co-located PCDs (Kane and Luz 2006a). But despite the impact of teleconference on the duration of a PCD, there is no significant difference in the overall diagnostic task structure between co-located discussions and those held in teleconference (Kane and Luz

\footnotetext{
12 Ziehl-Neelson method to colour mycobacteria with fuschin dye
} 
2006a). The relative duration for the diagnosis part of the PCD is almost $70 \%$ of the total time and this structure is relatively unaltered in teleconference (see Figure 5). Mean duration of combined D-Stages 1 and 2 is $74 \%$ and $67 \%$ for co-located and teleconference discussions, respectively. Analysis of vocalisation (talk spurt) patterns, reported in Kane and Luz (2006a), shows that the duration of an individual's vocalisation is significantly increased in teleconference and explains the extra time taken to conduct PCDs in teleconference. Nonetheless, the fact that discussion takes significantly more time in teleconference suggests that aspects of the technology impede, rather than support, awareness and interaction among the team when distributed across the teleconference interface.

Figure 5 also illustrates that the proportion of time taken in teleconference for D-Stages 1 and 2 is slightly shorter in teleconference than in co-located discussion, despite the overall discussion taking much longer in teleconference. The difference for D-Stage 1 is statistically significant using $t$-test (equal variance not assumed), calculated using the actual duration of the discussion in seconds, $t=-3.425$, $d f=44.55, p=0.001$. There is no statistical difference calculated for D-Stage 2 in teleconference. However, results suggest that a limitation of the teleconferencing technology among distributed team members may account for the differences observed.

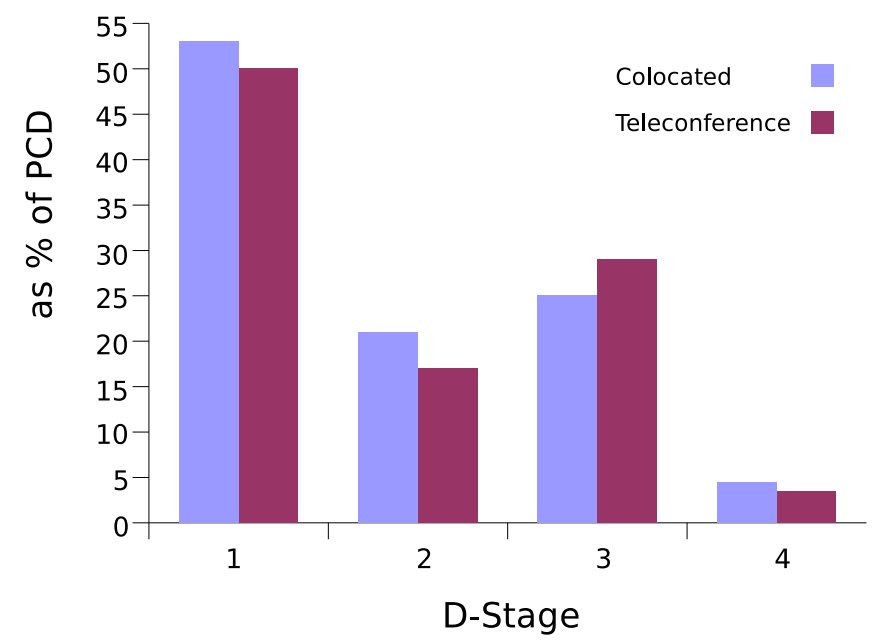

Fig. 5 Relative Mean duration of the diagnostic task (D-Stage 1 and 2) and the management decision task (D-Stages 3 and 4) in a PCD.

In teleconference, the remote participants are clinicians and present clinical findings across the teleconference interface. Radiology imaging studies and pathology specimens from remote hospitals are sent to the specialist centre, pre-MDTM, for review by the specialist radiologists and pathologists prior to discussion. During PCDs, the radiology and pathology presentation is controlled by the specialist centre and transmitted back to the remote 
participants. It is important that participants on both sides of a teleconference interface have access to the same level of image detail in any image presented ( $\mathrm{Li}$ et al. 2008). For some of the sites to which the main centre connects in teleconference, there is facility to transmit radiological imaging in teleconference. However, this facility is only used in the event of failure in the transmission of an image at the main centre. It has been found that the image quality across the teleconference interface is not of a high enough quality for expert opinion. Furthermore, it is recommended that specialists do not give professional opinion in discussion 'on the fly' without having had a considered review of the material, prior to the discussion, unless in exceptional circumstances (Board of the Faculty of Clinical Radiology 2005).

Analysis of the roles involved in D-Stage 1, when data is presented to the team, is illustrated in Table 1 . The relatively lengthy time spent reviewing radiology images presented by the radiologist reflects the importance of diagnostic imaging in patient investigations. Although radiology as a discipline is less specific than pathology, it is less invasive than surgical biopsy, carries less risk for patients and is more generally more acceptable. Values given on Table 1 are based on calculations of speech vocalisations from recordings of PCDs.

Table 1 Proportion of time spent by different roles during D-Stage 1 in an analysis of a set of 54 patient case discussions.

\begin{tabular}{lc} 
D-Stage 1 & $\begin{array}{c}\text { Contribution as Proportion (\%) } \\
\text { of Time taken in D-Stage 1 }\end{array}$ \\
\hline Respiratory Physician & 8.0 \\
Cardio-thoracic Surgeon & 6.9 \\
Medical Registrar & 13.9 \\
Surgical Registrar & 6.5 \\
Radiologist & 42.0 \\
Pathologist & 9.0 \\
Medical Oncologist & 2.7 \\
Nurse & 0.6 \\
Others & 10.3 \\
\hline
\end{tabular}

\section{Use of Artefacts, Gestures and Body Language}

At the meeting, team members sit facing in the same direction looking towards the main screen display. All participants, except the radiologist and pathologist, sit on plain chairs. The radiologist and pathologist sit in front on swivel chairs. They tend to sit sideways unless they are presenting images, in which case they have their backs to their 'audience'. They are observed to swivel fairly frequently towards speakers in the audience when in vocal interaction with team members. Otherwise they tend to gaze towards the image on the main display, like the other participants. For those sitting in plain chairs (not swivel), participants are observed to shift position from time to time, to observe a speaker, check orientation of an image, or consult the text 
display on the side wall (such as the name, or a clinical detail, of the patient being discussed). Most of the time they face the screen display, or may be seen reading from personal notes, or writing notes for later use.

The main artefacts used by the MDT at their meetings are those brought by the radiologist and pathologist to demonstrate their findings and explain the basis for their professional judgement. At the discretion of the clinician responsible for the case, images may be produced that were taken during bronchoscopy, or at surgery. In such cases the image will represent an unusual visual appearance, or highlight a particular difficulty encountered. In an unusual case, a radiation treatment plan may be shown to advise the team of the radiation treatment paths given to a patient, which might account for current unusual findings. Images from bronchoscopy are usually printed on paper, (as part of the bronchoscopy report) and shown to the group using the document imager. Movies or pictures from surgery are presented from a laptop projected onto the large screen display. These artefacts are used by individuals to illustrate a point with their fellow team members. While the images are most often used to enhance the professional opinion being offered, they serve as opportunities for peer review and tutorials. They also help provide understanding of the nature of the work of the individual specialists and the difficulties those individual team members can encounter in the course of their routine work.

\subsection{Radiology artefacts}

In radiology, images have traditionally been shared on analog film. With the advent of DICOM ${ }^{13}$ standards and $\mathrm{PACS}^{14}$ being implemented, digital imaging is often stored and shared electronically. However, the problem of old imaging (needed for review and comparison), and problems with compatibility between proprietary PACS systems and different digital imaging protocols between centres, have meant that images on film continue to be exchanged between hospitals. Films are typically viewed on a light box or by use of a document imager (shown in Figure 2). Problems using radiology film images are well documented, most notable the fact that up to $20 \%$ are missing when required (Strickland 2000). While electronic images facilitate simultaneous use by different users, there can be problems with network and performance. It has been observed that delays in loading electronic images at the MDTM can result in the radiologist deferring his contribution until later in the discussion (to allow time for images to load). A single access point to a PACS system can prove restrictive when several images need to be reviewed together that span a period of time. For instance, a pre- and post- treatment chest radiograph need to be reviewed together to establish if the treatment has had an effect on the original lesion which may often have subtle changes on imaging. It is reported that some radiologists prepare slide presentations of relevant imaging to MDTMs (Groth 2008), however for the group under study in this paper, it is preferred to have the full image sets available for

\footnotetext{
13 Digital Imaging and Communications in Medicine

14 Picture Archive and Communication System
} 
review during the patient case discussion. Preparing slide presentations preMDTM is time consuming prior to the meeting and while it will reduce time spent at the meeting in searching for specific images, the full image data is unlikely to be made available to the rest of the team for questioning in D-Stage 2. PACS allows for theoretically easy access to images, but it can be cumbersome. It requires navigation through a couple of screens to locate the required images, and images can be slow to load. Problems reported with PACS installations because of low image transfer and inadequate image quality (Inamura et al. 2001) can impact on MDTMs. Even when standards for individual viewing of radiological images may be satisfactory, speedy access is necessary to keep pace with the narrative account during MDTMs. While electronic images are accessed via the local area network (LAN), PET scans are always submitted on DVD, are loaded onto a PC and examined in a video clip. When played, the whole body image is rotated through 360 degrees, and an area of increased FDG avidity can be viewed in its 3-dimensional location.

\subsection{Pathology artefacts}

The pathologist usually brings microscope slides to the meeting to show the histological findings. During the presentation of patient imaging by the radiologist, the pathologist is often observed locating the feature to be demonstrated under the microscope. It is becoming a more popular and frequent practice for digital photographs to be taken during pathology pre-meeting preparation, and for those digital images to be taken on memory (USB) stick to the meeting, instead of the pathology slides. This practice saves meeting time and allows the pathologist to give full attention to the radiology presentation. Selection and annotation of tissue images prior to the MDTM for presentation during discussion, means that the pathologist more strictly determines the amount of tissue available to the MDT for review during the discussion. While the selected image may suffice for many cases, slide presentation of images are usually found to be most satisfactory to illustrate the gross appearance of a resected specimen on receipt in the laboratory and following pathology dissection, prior to tissue processing. Figure 6 shows a sample of pathology images that might be presented to an MDTM as a slide presentation.

Selection of microscopic tissue images for presentation to the MDTM does have a cost within the pathology department and is not always possible (Kane et al. 2007). As well as time shortage for the pre-MDTM review, sometimes the pathology sample may only be ready for microscopic assessment late on Friday afternoon and there is not time to prepare images in presentation format (such as the example on the right in Figure 6).

Radiologists and pathologists are regularly observed organising images during the initial presentation of the case (concurrent with interaction among other team members). 


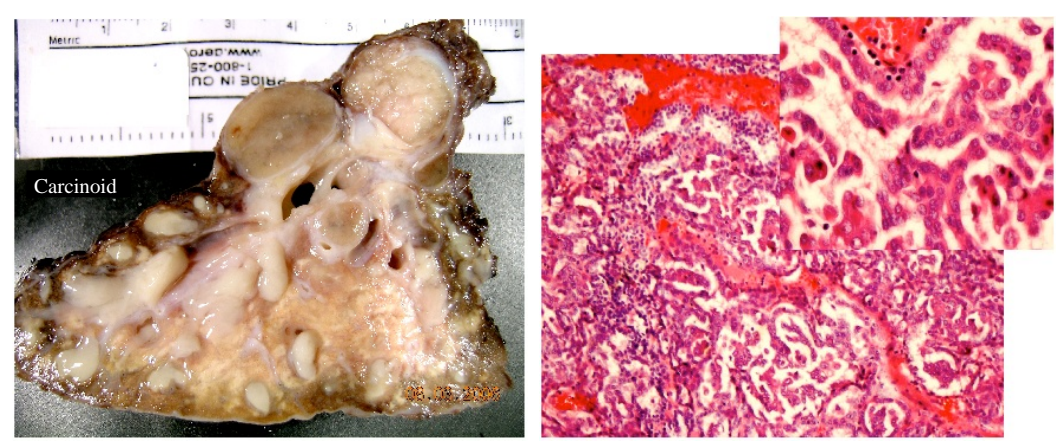

Fig. 6 Annotated image from gross tissue dissection in a pathology laboratory (left), and microscopic images of a tumour at different magnifications (right) presented to MDTM during discussion images used with permission

\subsection{Use of Gesture and Body Language}

Although participants sit facing forward, they are observed to attract one another's attention to detail in artefacts, using eye contact, gaze, head nods and finger gestures from time to time. When not discussing artefacts, participants tend to listen attentively and gaze at their personal notes, at the speaker, or even a blank monitor. Gazing at one another, and making eye contact is most notable when speech turns are being exchanged, a speaker wishes to engage another's attention, or a team member wishes to take the floor in discussion.

Pointing during D-Stage 1 is generally confined to the radiologist and pathologist when illustrating their findings. But others may gaze or point to the screen when asking a question about a feature, for example "what is that ...there ..." in D-Stage 2. Devices observed to point at a feature on the overhead display are given in Table 2:

Table 2 The variety of objects used as pointing and annotation devices during discussion

Technology in use, or Device used to draw attention, or focus,

\begin{tabular}{ll} 
audience participant & of team colleagues during discussion \\
\hline from document imager & pointer from graphics tablet, or pen, paper clip, \\
& or index finger \\
from computer & mouse \\
from microscope & laser pointer \\
from seat in audience & index finger or pen \\
& head nod and eye gaze
\end{tabular}

While pointers used with the document imager, and the mouse with a PC image, are visible to remote participants in teleconference, the use of a laser pointer (popular in co-located discussions) is not visible to remote parties. Also, when audience participants point or nod and gaze at the overhead screen, it is difficult to make these gestures visible in teleconference, since 
the teleconference link normally transmits the screen image, and not the room view when deictic references are being made.

Gesturing by seated participants is not very common but is occasionally observed in discussion on cases that are post-surgical resection, when the approach taken at operation is being described by the surgeon, particularly in cases of a difficult or unusual operation. Surgeons tend to use hand movements to describe the size, shape and orientation of a tumour at surgery and can be observed positioning and rotating their hands to demonstrate how they detached the tumour from the surrounding tissue. For example, when a pathologist shows an image of a resected specimen as in Figure 6 on page 25, the surgeon who removed it from the patient might use his/her hands to indicate the thickness of the tissue removed (which is not visible in the picture), or use gestures to describe its anatomical orientation, or fingers to indicate the distance from a cut surface from a major structure such as the heart, for example. Using representational gestures in this way seems to play a role in complementing the discussion with data which are not efficiently conveyed through other modalities such as speech and static graphics (Becvar et al. 2008). On failing to illustrate a point using hand gestures, surgeons have been observed to resort to using paper and pencil to draw the anatomical structures, and surgical margins ${ }^{15}$, to which they want to draw attention. Diagrams can help visualise the anatomical positioning of a tumour removed and help visualise the relationship between the cut surface in a pathology picture and the anatomical structures left behind in the patient. These types of discussions in D-Stage 2 usually take place when the surgical findings and pathology findings are being correlated.

Radiologists are occasionally observed to use their hands when describing how they orientated a patient when taking a fine needle aspiration ${ }^{16}$ (sampling method) or to estimate the three-dimensional size and shape of a tumour. Physicians who have undertaken a difficult bronchoscopy procedure have been observed to use their hands and fingers to describe the twists and turns needed to access particular segments of the lung, or to demonstrate their observations, while describing the anatomical location. It is as if the bronchoscopist visualises their experience when conducting the procedure and then proceeds to describe it, for instance:

"... endobronchially the airways are all splayed [stretching fingers] you can imagine - and compressed [drawing index finger and thumb together in a squeezing motion]. There is some kind of raggedness in the lower lobe ... the basal left lower lobe looked a bit abnormal, so ... we took various specimens."

\section{Discussion}

Establishing the definitive diagnosis for a patient is not an easy task and is often not straightforward (Symon et al. 1996). The diagnosis is established

\footnotetext{
${ }_{15}$ The cut edge of the tissue removed at operation is called the surgical margin 16 method to obtain a tissue sample for pathology, that is less invasive than surgical biopsy
} 
by gathering and sharing data among several specialists and interpreting these data within shared models of disease. The definitive diagnosis achieved through synchronous collaboration between clinical specialists, as described here, is considered to be a superior diagnosis with greater accuracy than the diagnosis that might be made by an individual clinician. There is opportunity for re-evaluation and refinement of opinion through contemporaneous interaction among the range of specialists involved in the data-gathering from the patient. It has been shown that making a diagnosis can be an iterative process (Cicourel 1990), that is refined through individual specialist interaction using questions, revising opinion and differentiation of features before a definitive diagnosis is reached in collaboration. The contemporaneous collaboration and pooling of information in the MDTM has a synergistic effect from which the hospital, the patient, the active participants and observers will benefit. Identifying how technology could improve specialist interactions and enhance awareness at MDTMs can be expected to deliver real benefits. Interaction between team members needs to be supported, and artefacts need to be easily handled, or manipulated, and useable for the different role purposes. As well as supporting the MDTM interactions, the information and decisions discussed at the MDTM need to be available to MDT members in their post-meeting tasks and responsibilities. The diagnosis agreed at the MDTM serves as a key reference item for subsequent interaction with the patient.

The description of the diagnostic work in PCDs in Section 4 illustrates particular roles on the team that have key tasks within the collaborative process. The PCD does not start from a position where all facts are made available to the team members equally. Radiologists and pathologists, as well as the clinician with responsibility for the patient, present patient data and occupy key roles in the discussion process. These expert-presenter roles, are in a privileged position since they have had prior access to the information and have had time to generate a considered opinion from the perspective of their speciality. They direct, or focus, the discussion since the information they present is ordered, and made available to other team members in the context of their experience. They are in a position to influence the discussion by the order in which they present the information, and the tone and language used in their presentation. Describing a patient as "this young woman of 65 , who complains of shortness of breath and ..." rather than "this elderly lady with shortness of breath, 65 years old, who ..." may invoke images of patients that may be quite different, even though the same data is presented in both cases.

Evaluation of the different specialist contributions to the PCD is reported elsewhere (Kane and Luz 2009) and it is shown that for those contributions that use images to support their findings, they rate more highly than those who articulate findings by voice alone. Figure 7 summarises responses from 91 active contributors to MDTMs and illustrates that for those specialities who rely on conveying clinical information through speech alone, such as communicating clinical findings on examination, they rate less favourably than those specialities who use images to support their opinion, such as pathology and radiology. Bronchoscopy findings are usually described in speech, but may 
be supported by photographs, from time to time, that were taken during a procedure. The use of images is also thought to increase the credibility of the presenter.

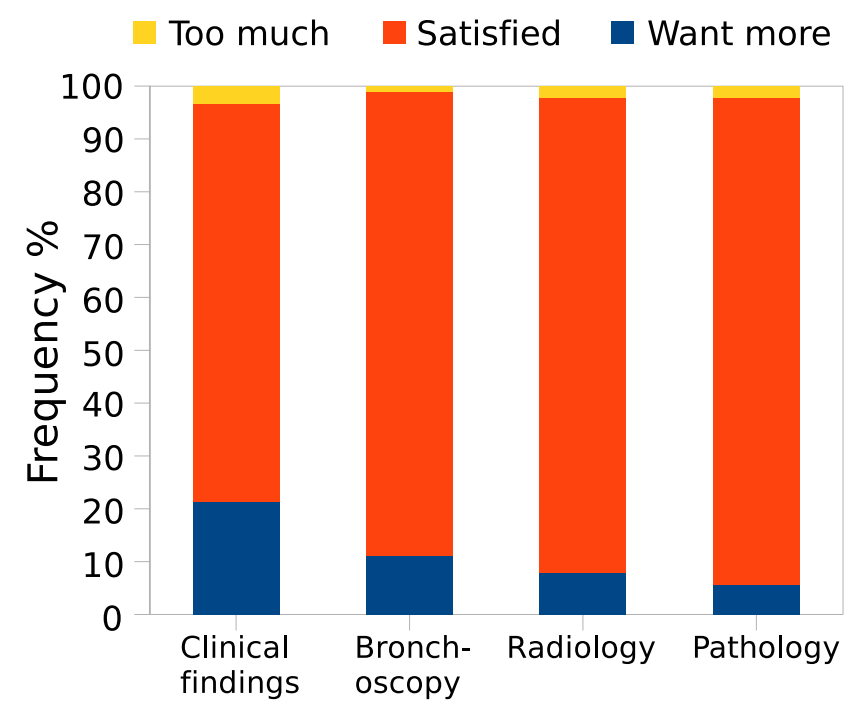

Fig. 7 Reported satisfaction with presentations from different roles to the MDTM, (from 91 active MDTM contributors). (from Kane and Luz (2009))

It is important for cohesiveness within the team that each role is proficient in their speciality and that their input supports the work of other specialties within the team. For the MDT to continue to be effective, all team members should attend the meetings and opinions are given in an open and respectful manner. The use of images to illustrate findings helps in the explanation of opinion expressed by a member of the team. As Goodwin (1994) noted, "As talk and image mutually enhance each other a demonstration that is greater than the sum of its parts emerges". Substantiating the underlying basis for the opinion offered, through the use of images, facilitates openness and transparency, and helps build trust among members of the team. Contributions at MDTM relies on voluntary participation. Thus, trust among team members is critical for the maintenance of a cohesive team, and for the continuance of the MDTM practice.

Conflict among decision-makers, particularly substantive conflict that involves opposition at the intellectual level, is shown to have positive effects upon group decisions by promoting better understanding of the issues involved (Lehto and Nah 2005). At the MDTM inconsistencies in findings between radiology, pathology and clinical staff are resolved through teasing out detail in discussion. It is rare for one of the specialities to maintain a minority position: the dissident opinion is usually revised in the light of the others' reports, or a decision is made to seek more data to resolve the question. 
Reaching consensus on the definitive diagnosis, or TNM disease staging in cancer patients, is the central tasks facing the MDT in discussion. None of the individual specialities have all of the information to make this decision. While the radiologist can estimate the size of the tumour, and the clinician can look for signs of advanced disease, and the pathologist can provide the tissue pathology, it is only by combining all three specialities will the full extent of the cancer be determined. Difficulties in distinguishing between a scenario where there are two independent small T1 tumours from a scenario with a single high grade tumour with lymph node disease, or metastases, can be difficult, and is very important. T1 tumours have the potential to be cured if surgically removed, while a patient with a T4 tumour is unlikely to be offered surgery as an option.

The provision of on-going feedback through regular audit is also important for team development. Through results of previous opinion offered, individual members of the team prove themselves, and over time, become 'tried and trusted' members of the team. It would be undermining for the team, for example, if the pathologist and radiologist disagree and it is left to the surgeon to establish which opinion was valid, post-resection. Individual credibility is built gradually over time. Reputation, or credibility, is usually based on previous contributions and communication behaviour within the team. The MDT develops in this way, as its members develop, and the team can gain or lose credibility both internally and external to the hospital.

The quality and standard of screens used for the display of artefacts deserves special mention. There are defined standards for the resolution of screens in radiology for diagnostic purposes, as radiological diagnosis relies on fine-grained detail to differentiate between anatomical structures. For pathology, the visual display should support the resolution needed to demonstrate fine microscopic detail while maintaining colour definition. (Dye chemistry is used to differentiate in histological structures.) Unfortunately the screens and equipment often provided in meeting rooms is considered to be of 'teaching' quality rather than 'diagnostic' quality. While a teaching level quality may be satisfactory in most respects, it is difficult for the radiologist and pathologist, and potentially undermining, if they try to describe a detail that is not visible to the group at the MDTM. Radiologists and pathologists are most reluctant to offer opinion on images transmitted across a teleconferencing interface, mainly because they give a considered opinion and do not like to be asked to give an 'on-the-spot' diagnosis. In fact, it is ill-advised, and not in the best interest of patients, for radiologists to provide on-the-spot diagnosis (Board of the Faculty of Clinical Radiology 2005). These professionals are also reluctant to give an immediate opinion in teleconference, because the quality of transmitted image date tends to be of teaching quality rather than diagnostic quality, with less resolution of fine detail. The refined perceptual skill of pathologists and radiologists, in particular, has been developed with technological support that allows fine-grained resolution of detail. These specialists exhibit 'professional vision' described by Goodwin (1994) whereby i) phenomena are coded and transformed into objects of knowledge, ii) specific phenomena are highlighted in a complex perceptual field and iii) material representations are produced and articulated. This situation has parallels with 
the observation by Harper and Sellen (1995) that information that requires a high degree of professional judgement in its production may be difficult to support through groupware.

The provision of quality data is central to a satisfactory PCD. In an attempt to support more data being available, and also to provide an awareness mechanism, the group designed a template that provides text data on display during the PCD. The template displays the patient's critical identifiers, namely name, date of birth, and hospital number, as well as the clinician responsible for the case and key items of information, such as the patient's smoking history and clinical summary of the case. The text display serves as a reminder, or prompt, for the patient who is currently being discussed, as well as making available known information anticipated to be required in the discussion. This text display is located on a side wall in the meeting room (illustrated in Figure 2), and is projected onto the Smartboard ${ }^{\mathrm{TM}}$ with a ceiling projector. The quality of the image from the projector is satisfactory for the text display, but is not suitable for image projection because of a loss of resolution and colour distortion in projected images.

\subsection{Time and timing}

The timing of the independent related processes is critical for a successful PCD, and to have a satisfactory diagnosis. Each process must work efficiently within a limited timeframe. Pathology, for example, is a necessary part of the diagnostic task and processes must be completed in order for the tissue features to be incorporated into the definitive diagnosis agreed at the PCD. In fact without a tissue diagnosis, it is not possible to establish a disease stage.

Time is also important with respect to the patient investigations. A number of events will have occurred, over time, that contribute to the diagnosis. Symptoms may have lessened or got worse, for example. At the MDTM several radiological images are reviewed and the time frame between image sets is important. It is a requirement that images can be compared over time. Yet, often only one monitor is available for reviewing electronic images and most PACS do not allow for more than one image window to be open at one time. Currently teams utilise the document imager and two PCs to move between hard copy film, and images from CD or PACS.

The additional time cost of discussions in teleconference has significant implications for the development of tele-facilitated services over geographical distance. Given the time constraints in a busy hospital, finding time for all of the team members to meet within individual schedules is difficult. The additional time required to engage in discussions across a teleconference has the potential to undermine the specialist services concentrated at the large hospital, and can be frustrating for remote team members who would like to spend more time in discussion Kane et al. (2007). Although the technology has benefits for the patients (who don't have to travel to the specialist centre for assessment), there is little benefit for those using the technology, and in fact, there is a real time cost involved. 
In providing technological support for the MDTM, it needs to be recognised that the task of diagnosis through collaboration is one of the main functions of the meeting. However, for any solution to be satisfactory, account must be taken of the secondary educational, organizational and social functions of the discussion, including team development.

While tools that would directly support collaboration are needed, such as, pointing devices and tools for drawing and annotation, more powerful systems that will allow for the rapid manipulation of large image data sets are needed for the full benefits of this sort of collaborative practice to be realised.

The practice of collaborative definitive diagnosis is expected to develop for the foreseeable future. This will pose special challenges for electronic patient record keeping. Questions that are being addressed include: 'what sort of record is needed?', 'do we include specific images?', 'do we need to document the differential diagnosis considered?', 'what if there is a disagreement? Should the dissenting opinion be included too?'. In the development of future electronic support for collaboration and record keeping, innovative and novel solutions will need to be developed.

\section{Conclusion}

Diagnosis by consensus at MDTMs is of special interest to CSCW researchers, in that it is (a) interactive with respect to presentation and interpretation of data and information and (b) it helps to build a shared awareness of the diagnosis and decisions that depend on it. Any technology that affects interaction and awareness will thus affect diagnostic work undertaken by a multidisciplinary team.

Achieving a diagnosis, by consensus, through a collaborative process that utilises independent investigative data gathering, and involves many specialities, is likely to yield a more reliable diagnosis than one achieved by a lone diagnostician. In this respect, the MDTM can be regarded as a high performance work system. Providing the wide range of support needed for such collaborative activities poses a challenge for developers. Technologies that support individual professionals in their use of large image data, and also support groups interacting though dialogue, to provide a definitive diagnosis, need to be integrated so that professionals can access and talk about the fine-grained detailed information necessary for diagnosis, in a speedy and visible manner. Many areas of improvement can be identified which range from low-tech solutions such as the placement of microphones, to the use of novel technologies such as mobile and ubiquitous computing. Pointing and annotation devices, as well as a meeting record and decision support tools, have potential to improve MDTM services. While the diagnostic task is foremost in mind in this account of MDTM work, it must also be remembered that this forum satisfies many organizational and social functions, in addition to its patient management role. As well as improved decision-making, with ownership and responsibility, increasing cross disciplinary co-operation and understanding, peer review and support (including teambuilding) are recognised benefits of MDTMs (Ruhstaller et al. 2006). Teamwork is more 
successful when decision-making is visible and participative (Ruhstaller et al. 2006). For technological solutions to be effective, they must be able to fulfil the range of functions served through the group activity.

Poor communications and failures in interpersonal interaction can account for 70-80\% of errors in healthcare (Arnaudova and Jakubowski 2005) and the MDTM is a forum where good communication among specialists is of critical importance. It is explained in section 4 how team members engage in rapid speech exchange, use images and gestures to illustrate their opinion and rely on a PACS, that is located elsewhere. It is also a requirement that cancer patients are managed through these meetings and that all the necessary specialists are present, and participate as appropriate to their service specialty, the quality of the discussion and needs of the patient. Thus, the ideal room for the MDTM will have a high speed wireless network, utilise ubiquitous devices to record the presence of individuals, will maximise the visual display area potential of the space and have enhanced audio support. The room would be equipped with enough visual display area to allow the comparison of several images at once, and allow for the simultaneous display of the bronchoscopy image, microscopic image and/or a video taken in the operating theatre. A record, or outcome, of the discussion will be available for review, as needed, afterwards.

Differences in the levels of interaction and awareness of process among participants in teleconference have deep implications for policy makers who may wish to concentrate specialist medical services in a small number of centres and deliver medical service to a large geographical area via teleconferencing technology (Huh et al. 2000). Results presented here suggest that large scale practice of conducting PCDs over a teleconference network is unsustainable using the technology that is currently available.

Diagnosis by consensus is now recognised as a high performance model for clinical decision making, and we can expect that the practice of MDTMs will continue to grow for the foreseeable future. While this model of team working has been developed in healthcare, we anticipate that it will adopted in a wider range of industries and services once its benefits become evident in research.

Acknowledgements Our thanks to Dr. Finbarr O'Connell and all the members of the multidisciplinary team at the respiratory MDTM in St James's Hospital, Dublin, for their co-operation in this on-going study. We also thank Dr. Nicholson for use of the photographs, and the anonymous reviewers for this paper for their constructive comments. The co-operation of the Division of Radiation Therapy, Trinity College Medical School, is gratefully acknowledged. This research is supported by funding by the SFI Research Frontiers grant under the National Development Plan.

\section{References}

AJCC: 1992, 'Manual for Cancer Staging'. American Joint Committee on Cancer, 4 th edition.

Alberts, W. M.: 2007, 'Diagnosis and Management of lung cancer executive summary: ACCP Evidence-based clinical practice guideline'. Chest 132(3 supplement), 1-19. 
Alberts, W. M., G. Bepler, T. Hazelton, J. C. Ruckdeschel, and J. H. Williams: 2003, 'Practice Organization'. Chest 123(1 Supplement), 332S - 337S.

Arnaudova, A. and E. Jakubowski: 2005, Eight Futures Forum on governance of patient safety. WHO, Copenhagen, Denmark.

Balding, C. and J. Anderson: 2007, 'Clinical excellence in cancer care. A model for safety and quality in Victorian cancer services.'. Technical report, Metropolitan Health and Aged Care Services Division, Victorian Government, Australia.

Batchelor, I. and J. McFarlane: 1980, Multi-Disciplinary Clinical Teams. Based on working papers of the Royal Commission on the NHS, King's Fund, RC12.

Becvar, A., J. Hollan, and E. Hutchins: 2008, 'Representational Gestures as Cognitive Artifacts for Developing Theories in a Scientific Laboratory'. In: Resources, Co-Evolution and Artifacts: Theory in CSCW. pp. 117-143. 10.1007/978-184628-901-9_5.

Berg, M.: 1992, 'The construction of medical disposals. Medical sociology and medical problem solving in clinical practice'. Sociology of Health and Illness 14(2), 151-180.

Birchall, M., D. Bailey, and P. King: 2004, 'Effect of process standards on survival of patients with head and neck cancer in the south and west of England'. British Journal of Cancer 91(8), 1477-1481.

Board of the Faculty of Clinical Radiology: 2005, Cancer Multidisciplinary Team Meeting - Standards for Clinical Radiologists. London.

Calman, K. and D. Hine: 1995, A Policy Framework for Commissioning Cancer Services. Department of Health, Welsh Office.

Chin, R., T. W. McCain, M. A. Lucia, J. O. Cappellari, N. E. Adair, J. F. Lovato, D. P. Dunagan, M. A. Brooks, H. P. Clark, and E. F. Haponik: 2002, 'Transbronchial needle aspiration in diagnosing and staging lung cancer: how many aspirates are needed?'. American Journal of Respiratory and Critical Care Medicine 166(3), 377-381.

Cicourel, A. V.: 1990, 'The integration of distributed knowledge in collaborative medical diagnosis'. In: J. Galegher, R. E. Kraut, and C. Egido (eds.): Intellectual Teamwork: Social and Technological Foundations of Co-operative Work. Hillsdale, New Jersey: Lawrence Erlbaum Associates, pp. 221-242.

Elstein, A. S. and A. Schwarz: 2002, 'Clinical problem solving and diagnostic decision making: selective review of the cognitive literature'. British Medical Journal 324, 729-732.

Gallagher, E. J.: 2003, 'The Problem with Sensitivity and Specificity'. Annals of Emergency Medicine 42(2), 298-303.

Genest, C. and J. V. Zidek: 1986, 'Combining Probability Distributions: A Critique and an Annotated Bibliography'. Statistical Science 1(1), 114-135.

Goodwin, C.: 1994, 'Professional vision'. American Anthropologist 96(3), 606-633.

Groth, K.: 2008, 'The Role of the Technology in Video-Mediated Consensus Meetings'. In: TTeC 2008. Trømsø, Norway, Trømsø Telemedicine Laboratory.

Guyatt, G. H., R. B. Haynes, R. Z. Jaeschke, D. J. Cook, L. Green, C. D. Naylor, M. C. Wilson, and W. S. Richardson: 2000, 'Users' Guides to the Medical Literature: XXV. Evidence-based medicine: principles for applying the Users' Guides to patient care. Evidence-Based Medicine Working Group.'. Journal of the American Medical Association 284(24), 3127-3128.

Haidet, P. and D. A. Paterniti: 2003, "Building" a History Rather than "Taking" One'. Archives of Internal Medicine 163, $1134-1140$.

Hall, P. and L. Weaver: 2001, 'Interdisciplinary education and teamwork: a long and winding road'. Medical Education 35(9), 867-875.

Hardstone, G., M. Hartswood, R. Proctor, R. Slack, and A. Voss: 2004, 'Supporting Informality: Team working and Integrated Care Records'. In: CSCW Chicago 2004. ACM Press.

Harper, R. H. R. and A. Sellen: 1995, 'Collaborative Tools and the Practicalities of Professional Work at the International Monetary Fund'. In: CHI. pp. 122-129.

Hogarth, R. M.: 1986, 'Comment on: 'Combining probability distributions: A critique and an annotated bibliography". Statistical Science 1(1), 145-147. 
Hollywood, D.: 2007, 'TNM staging with metastases'. Personal Communication August 20th, 2007.

Houssami, N. and R. Sainsbury: 2006, 'Breast cancer: Multidisciplinary care and clinical outcomes'. European Journal of Cancer 42, 2480-2491.

Huh, S. J., H. Shirato, S. Hashimoto, S. Shimizu, D. Y. Kim, Y. C. Ahn, D.-R. Choi, K. Miyasaka, and J. Mizuno: 2000, 'An integrated service digital network (ISDN)-based international telecommunication between Samsung Medical Center and Hokkaido University using telecommunication helped radiotherapy planning and information system (THERAPIS)'. Radiotherapy and Oncology $\mathbf{5 6}(1), 121-123$.

Inamura, K., J. Konishi, H. Nishitani, S. Kousaka, Y. Matsumura, H. Takeda, and H. Kondoh: 2001, 'Status of PACS and technology assessment in Japan'. Computer Methods and Programmes in Biomedicine 66, 5-15.

Jefford, M., R. Jennens, T. Speer, and V. Thursfield: 2007, 'Different professionals' knowledge and perceptions of the management of people with pancreatic cancer.'. Asia-Pacific Journal of Clinical Oncology 3, 11-51.

Jordan, B. and A. Henderson: 1995, 'Interaction Analysis: Foundations and Practice'. The Journal of the Learning Sciences 4(1), 39 - 103.

Kane, B. and S. Luz: 2006a, 'Multidisciplinary Medical Team Meetings: An Analysis of Collaborative Working with Special Attention to Timing and Teleconferencing'. Computer Supported Co-operative Work (CSCW) 15(5-6), 501 535.

Kane, B. and S. Luz: 2006b, 'Probing the Use and Value of Video for MultiDisciplinary Medical Teams in Teleconference'. In: Proceedings of the 19th IEEE International Symposium on Computer-Based Medical Systems. pp. 518523, IEEE Computer Society.

Kane, B. and S. Luz: 2009, 'Information sharing at multidisciplinary medical team meetings'. Group Decision and Negotiation In press.

Kane, B., S. Luz, and M. Buckley: 2008, 'Multidisciplinary team working and changing care pathways: Challenging traditional models of electronic record keeping.' In: Health Informatics Society of Ireland. Dublin, Ireland, HISI.

Kane, B., S. Luz, D. S. O'Briain, and R. McDermott: 2007, 'Multidisciplinary team meetings and their impact on work-flow in Radiology and Pathology Departments'. BMC Medicine 5(15).

Katterhagen, J. G. and D. L. Wishart: 1977, 'The Tumor Board - How It Works in a Community Hospital'. Ca A Cancer Journal for Clinicians 27(4), 201-204.

Lababede, O., M. A. Meziane, and T. W. Rice: 1999, 'TNM Staging of Lung Cancer, a quick reference chart'. Chest 115, 233-235.

Lehto, M. R. and F. Nah: 2005, 'Decision-making models and decision support'. In: G. Salvendy (ed.): Handbook of Human Factors and Ergonomics. New York: Wiley.

Li, J., T. Mansfield, and S. Hansen: 2008, 'Supporting Enhanced Collaboration in Distributed Multidisciplinary Care Team Meetings'. In: 21st IEEE International Symposium on Computer-Based Medical Systems. Jyväskylä, Finland, pp. 482-487, IEEE.

Mott, B., C. Callaway, L. Zettlemoyer, S. Lee, and J. Lester: 1999, 'Towards narrative-centered learning environments'.

National Collaborating Centre for Acute Care (NICE): 2005, Diagnosis and treatment of lung cancer. Methods, Evidence and Guidance. London.

NCCAC: 2005, The diagnosis and treatment of lung cancer. Vol. CG24. NICE.

Nicholls, J.: 2007, 'Editorial: The inflammatory bowel disease unit and the multidisciplinary team meeting'. Colorectal Disease 9, 477.

O'Higgins, N.: 2006, Developing Quality Care for Breast Services in Ireland.

Ontario, C. C. O. A. C.: 2008, 'Multidisciplinary cancer conferences'.

Øvretveit, J.: 1999, Integrated quality development for public healthcare. Norwegian Medical Association.

Paternò, F.: 2000, Model-Based Design and Evaluation of Interactive Applications, Applied Computing. Springer-Verlag. 
Pinelle, D., C. Gutwin, and S. Greenberg: 2003, 'Task analysis for groupware usability evaluation: Modeling shared-workspace tasks with the mechanics of collaboration'. ACM Trans. Comput.-Hum. Interact. 10(4), 281-311.

Randall, D., R. Harper, and M. Rouncefield: 2007, Fieldwork for Design. Springer.

Rasmussen, P. C. and S. Bulow: 2005, 'FFuture organisation of colorectal cancer surgery in Denmark]'. Ugeskr Laeger 167(44), 4191-2.

Richardson, W. S., M. C. Wilson, G. H. Guyatt, D. J. Cook, and J. Nishikawa: 1999, 'Users' guides to the medical literature: XV. How to use an article about disease probability for differential diagnosis. Evidence-Based Medicine Working Group'. Journal of the American Medical Association 281(13), 1214-1219.

Ruhstaller, T., H. Roe, B. Thürlimann, and J. J. Nicholl: 2006, 'The Multidisciplinary meeting: An indispensable aid to communication between different specialities'. European Journal of Cancer 42, 2459-2462.

Sainsbury, R., B. Haward, L. Rider, C. Johnston, and C. Round: 1995, 'Influence of clinician workload and patterns of treatment on survival from breast cancer'. Lancet 345, 1265-1270.

Strickland, N. H.: 2000, 'Current topic: PACS (picture archiving and communication systems): filmless radiology'. Arch Dis Child 83(1), 82-86.

Symon, G., K. Long, and J. Ellis: 1996, 'The Co-ordination of Work Activities: Co-operation and Conflict in a Hospital Context'. Computer Supported Cooperative Work (CSCW) $\mathbf{5}(1), 1-31$.

van der Veer, G., B. Lenting, and B. Bergevoet: 1996, 'GTA: Groupware Task Analysis - Modeling Complexity'. Acta Psychologica 91, 297-322.

Weller, S. C. and N. C. Mann: 1997, 'Assessing Rater Performance without a "Gold Standard" Using Consensus Theory'. Medical Decision Making 17(1), 71-79.

Windsor, A. and A. Forbes: 2007, 'The inflammatory bowel disease unit and the multidisciplinary team meeting'.

Wit, J.: 1998, 'Rational Choice and the Condorcet Jury Theorem'. Games and Economic Behavior 22(2), 364-376.

Wong, K. and D. Birks: 2004, 'Surgical risk management: the value of a weekly surgical radio-pathological meeting'. Anz J Surg 74(4), 205-209.

Wright, F. C., C. De Vito, C. Langer, A. Hunter, and the Expert Panel on Multidisciplinary Cancer Conference Standards: 2007, 'Multidisciplinary cancer conferences: A systematic review and development of practice standards'. European Journal of Cancer 43, 1002-1010. 